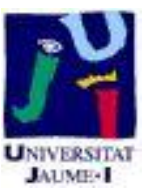

Título artículo / Títol article: Diffusion-Recombination Impedance Model for Solar Cells with Disorder and Nonlinear Recombination

Autores / Autors

Bisquert, Juan ; Mora Seró, Iván ; Fabregat Santiago, Francisco

Revista:

ChemElectroChem, 2014, vol. 1, no 1

Versión / Versió:

Postprint del autor

Cita bibliográfica / Cita bibliogràfica (ISO 690):

url Repositori UJI:
BISQUERT, Juan; MORA - SERO, Iván;

FABREGAT - SANTIAGO, Francisco. Diffusion-

Recombination Impedance Model for Solar Cells with Disorder and Nonlinear Recombination.

ChemElectroChem, 2014, vol. 1, no 1, p. 289-296

http://hdl.handle.net/10234/91811 


\title{
Diffusion-Recombination Impedance Model for Solar Cells with Disorder and Nonlinear Recombination
}

\author{
Juan Bisquert, ${ }^{*}$ Iván Mora-Sero, and Francisco Fabregat-Santiago ${ }^{[a]}$
}

The diffusion-recombination model is a key tool in understanding the photovoltaic operation of solar cells. Dye-sensitized solar cells, organic solar cells, and inorganic semiconductor solar cells are systems affected by disorder that are often characterized with impedance spectroscopy. In this paper, we extend the previous theory of diffusion-recombination impedance including traps and nonlinear recombination. We show the transmission line equivalent circuit representation, and we describe the physical meaning of a number of model parameters that can be obtained: the chemical capacitance, $C_{\mu}$; the re- combination resistance, $R_{\text {reci }}$ the transport resistance, $R_{\mathrm{tr}} ;$ the electron lifetime, $\tau_{n}$; the electron conductivity, $\sigma_{n}$; the chemical diffusion coefficient of electrons, $D_{n}$; and the diffusion length, $L_{n}$. At most, three of these parameters are independent, but if the diffusion length is short, the impedance model collapses to a function that has one degree of freedom less, known as the Gerischer impedance. We show the connection of the two parameters that remain to the diffusion length and the lifetime.

\section{Introduction}

The diffusion-recombination impedance model ${ }^{[1]}$ has been widely applied to the characterization of solar cells by using the technique of impedance spectroscopy. ${ }^{[2-4]}$ Since its original derivation, ${ }^{[1]}$ a number of additional physical features have appeared, mainly in the study of dye-sensitized solar cells (DSCs), ${ }^{[5]}$ but also in the study of other systems that were not treated in the model. First is the predominance of traps in disordered materials, which strongly influences the measured kinetic coefficients. This issue was first solved by using the quasistatic approximation, ${ }^{[6,7]}$ which modifies the time constants associated to the transport level with a trapping factor. A full theory that rationalizes the interpretation of measured coefficients and their relation to stochastic simulations was formulated thereafter. ${ }^{[5,8,9]}$ In addition, it was shown that nonlinear recombination is the rule in systems such as DSCs and organic solar cells, ${ }^{[3,10,11]}$ and such a nonlinear feature enhances inhomogeneous behavior. ${ }^{[12]}$

In this paper, we provide a more general derivation of the impedance model that includes the trapping factors and that uses a nonlinear recombination rate. The equations used to determine the impedance are formulated, and they are solved analytically for the homogeneous and linear cases. This general formulation reveals the factors that introduce local variations, which, in addition to nonlinear recombination, requires a numerical solution method.

[a] Prof. J. Bisquert, Dr. I. Mora-Sero, Dr. F. Fabregat-Santiago

Photovoltaics and Optoelectronic Devices Group

Departament de Física, Universitat Jaume I

12071 Castelló (Spain)

E-mail: bisquert@uji.es

Supporting Information for this article is available on the WWW under http://dx.doi.org/10.1002/celc.201300091.
The most widely used case in applications is that in which the diffusion length, $L_{n}$, is longer than the active layer thickness, $L$, as usually found, for example, in DSCs. ${ }^{[2,13-15]}$ However, in other instances the diffusion length could become shorter than $L$, and in that case the general diffusion-recombination impedance formula converges to the Gerischer impedance, in which the number of parameters is reduced by one with respect to the former. This situation has been recognized by some authors, ${ }^{[16]}$ but it is of considerable interest to clarify the interpretation of the remaining parameters from a theoretical point of view. We will derive explicit parameters of the Gerischer impedance and their connection to the lifetime and diffusion length.

\section{Kinetic Model and Interpretation of Kinetic Constants}

We consider the diffusion-recombination model in the presence of traps, in a 1D slab of area $A$. We denote $n_{\mathrm{c}}$ as the carrier density in the transport level, $n_{\mathrm{L}}$ as the carrier density in localized band-gap states, and $n=n_{\mathrm{c}}+n_{\mathrm{L}}$ as the total carrier density. The model can be formulated in terms of the following conservation equation [Eq. (1)]:

$\frac{\partial n_{\mathrm{c}}}{\partial t}=G(x)-\frac{\partial J_{n}}{\partial x}-U_{n}\left(n_{\mathrm{c}}\right)-\frac{\partial n_{\mathrm{L}}}{\partial t}$

in which $J_{n}$ is the electron flux, related to the free-electron diffusion coefficient, $D_{0}$, by Fick's law [Eq. (2)]:

$J_{n}=-D_{0} \frac{\partial n_{\mathrm{c}}}{\partial x}$ 
In Equation (1), $G$ is a local generation rate, and $U_{n}$ is the recombination rate per unit volume. At the blocking boundary $J_{n}(x=L)=0$. At the extraction contact, the carrier density is controlled by the voltage. The voltage $V$ is given by the increase in the Fermi level of the electrons relative to the Fermi level of the hole carriers, which is considered stationary at the dark equilibrium value $E_{\mathrm{F} 0}$ [Eq. (3)]:

$q V=E_{\mathrm{Fn}}(x=0)-E_{\mathrm{F} 0}$

In this equation, $q$ is the positive elementary charge. In Equation (1), we adopt a multiple trapping model that has been shown by experiment and simulation to describe the characteristics of disordered systems. In many cases, hopping systems can also be reduced to the multiple trapping model by using the concept of transport energy. $[8,9,17]$

Next, we introduce some magnitudes that enter the expression of impedance. ${ }^{[4]}$ First, the chemical capacitance is defined as follows [Eq. (4)]: ${ }^{[18]}$

$C_{\mu}=L A q \frac{d n}{d V}$

A specific chemical capacitance per unit volume is defined as [Eq. (5)]:

$c_{\mu}=q \frac{d n}{d V}$

In the literature, we find two main types of chemical capacitance. The conduction band capacitance is obtained as [Eq. (6)]:

$c_{\mu}^{\mathrm{cb}}=q \frac{d n_{\mathrm{c}}}{d V}=\frac{n_{\mathrm{c}} q^{2}}{k_{\mathrm{B}} T}$

in which $k_{\mathrm{B}} T$ is the thermal energy. The capacitance of the traps is associated, within good approximation, to the density of states of the localized states, $g(E)$, calculated at the Fermi level [Eq. (7)]:

$c_{\mu}^{\text {trap }}=q \frac{d n_{L}}{d V_{\mathrm{F}}}=q^{2} g\left(E_{\mathrm{F} n}\right)$

In practice, it is often found that $c_{\mu}^{\text {trap }} \gg c_{\mu}^{\mathrm{cb}}$, so that $c_{\mu}^{\text {tot }}=c_{\mu}^{\text {cb }}+c_{\mu}^{\text {trap }} \approx c_{\mu}^{\text {trap }}$.

We also introduce the recombination resistance, which is given by the reciprocal derivative of recombination flux [Eq. (8)]:

$R_{\mathrm{rec}}=\frac{1}{A}\left(\frac{\partial j_{\mathrm{rec}}}{\partial V}\right)^{-1}$

The flux depends on the volume recombination rate as [Eq. (9)]: $j_{\text {rec }}=q L U_{n}$

The electron lifetime is given by [Eq. (10)]: ${ }^{[19]}$

$\tau_{n}=\left(\frac{\partial U_{n}}{\partial n}\right)_{\bar{n}}^{-1}$

Therefore, the following is obtained [Eq. (11)]:

$R_{\text {rec }}=\frac{\tau_{n}}{C_{\mu}^{\text {tot }}}$

Another important quantity is the transport resistance, given by the expression [Eq. (12)]:

$R_{\mathrm{tr}}=\frac{L}{A \sigma_{n}}$

in terms of the electron conductivity [Eq. (13)]:

$\sigma_{n}=\frac{q^{2} D_{0} n_{\mathrm{c}}}{k_{\mathrm{B}} T}=D_{0} c_{\mu}^{\mathrm{cb}}$

It is useful to introduce an alternative expression for the conductivity, given by the generalized Einstein relation, that links the conductivity, the chemical diffusion coefficient, and the chemical capacitance [Eq. (14)] ${ }^{[8]}$

$\sigma_{n}=D_{n} c_{\mu}^{\text {tot }}$

Therefore [Eq. (15)]:

$R_{\mathrm{tr}}=\frac{L}{A D_{n} C_{\mu}^{\mathrm{tot}}}=\frac{L}{A D_{0} C_{\mu}^{\mathrm{cb}}}$

We should remark that $D_{n}$ is the chemical diffusion coefficient, which is measured by any small perturbation technique. Introducing the trapping factor [Eq. (16)]:

$\Theta_{L}=\left(1+\frac{\partial n_{L}}{\partial n_{c}}\right)=\frac{c_{\mu}^{\text {tot }}}{c_{\mu}^{c b}}$

we have [Eq. (17)]:

$D_{n}=\frac{1}{\Theta_{L}} D_{0}$

Similarly, the measured lifetime is given by Equation (10), and one can also define the lifetime of the free carrier, $\tau_{f}$ [Eq. (18)]:

$\tau_{f}=\left(\frac{\partial U_{n}}{\partial n_{c}}\right)_{\bar{n}_{c}}^{-1}$

which relates to the lifetime of the electron as [Eq. (19)]:[9, 19] 
$\tau_{n}=\Theta_{L} \tau_{f}$

Therefore, we can write [Eq. (20)]:

$R_{\text {rec }}=\frac{\tau_{f}}{C_{\mu}^{\mathrm{cb}}}$

which is equivalent to Equation (11).

In general, we observe that there are two sets of quantities: those that are for free carriers, $D_{0}, \tau_{0}$, and $c_{\mu}^{c b}$, and those that include free and trapped carriers, $D_{n}, \tau_{n}$, and $c_{\mu}^{\text {tot }}$. Sometimes we can express impedance parameters in terms of either set, as shown in Equations (11), (15), and (20). It should be emphasized that some impedance elements effectively depend only on the free carriers, as in Equations (15) and (20). However, below we show that $c_{\mu}^{\text {tot }}$, including the capacitance of the traps, also appears in the impedance functions, which is indicative of the fact that we cannot get rid of the traps. Therefore, small perturbation techniques that provide kinetic coefficients, such as intensity-modulated photocurrent spectroscopy (IMPS), intensity-modulated voltage spectroscopy (IMVS), and time transient decays, always give $D_{n}$ and $\tau_{n}$ and not the free-carrier counterparts. The interpretation of these kinetic constants is completed with single particle quantities such as the jump diffusion coefficient, $D_{J}$, which appears explicitly in stochastic sim-

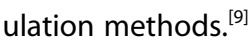

The diffusion length is given by [Eq. (21)]:

$L_{n}=\sqrt{D_{n} \tau_{n}}$

In the multiple trapping model, the trapping factors $\Theta_{L}$ compensate for the diffusion length. ${ }^{[6]}$ However, if the lifetime of the free carrier shows some dependence on the potential, as implied by Equation (18), then the diffusion length varies with voltage, according to [Eq. (22)]:[12]

$L_{n}=\sqrt{D_{0} \tau_{f}}$

This feature is often observed in experimental results. ${ }^{[13,20]}$ In the measurements of DSCs, it is often observed that the diffusion length increases as the voltage increases due to the increase in the lifetime of the free carrier. ${ }^{[19,21,22]}$ However, the present theory applies equally well to either an increase or a decrease in $L_{n}$. The connection of the diffusion length with the trap dynamics was also investigated by Monte Carlo simulation. ${ }^{[17,23]}$

In nanostructured and porous systems, the above quantities that depend on the volume of the system should be modified by the porosity, $p$, of the layer. The steady-state concentration obtained by solution of Equation (1) is shown in Figure 1 for different values of the diffusion length.

On the basis of the above-introduced kinetic constants for transport and recombination, we can define the characteristic angular frequency of recombination [Eq. (23)]:

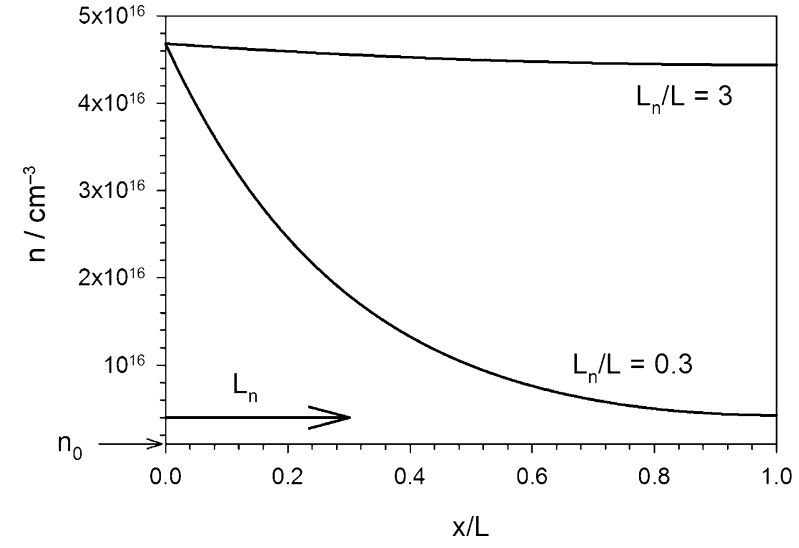

Figure 1. Distribution of electrons injected by the applied voltage to a semiconductor layer at $x=0$ with a blocking boundary condition at $x=L$ for different values of the diffusion length $L_{n}$. The baseline concentration $n_{0}$ is indicated.

$\omega_{\text {rec }}=\tau_{n}^{-1}$

and the characteristic angular frequency for the transit time [Eq. (24)]:

$\omega_{\mathrm{d}}=\frac{D_{n}}{L^{2}}$

We note the factor relating the characteristic frequencies, which can be expressed in several alternative ways [Eq. (25)]: ${ }^{[1]}$

$\frac{\omega_{\mathrm{d}}}{\omega_{\mathrm{rec}}}=\frac{R_{\mathrm{rec}}}{R_{\mathrm{tr}}}=\left(\frac{L_{n}}{L}\right)^{2}$

\section{Calculation of Diffusion-Recombination Impedance in Multiple Trapping}

The calculation of the impedance requires the determination of the quotient of current to voltage under small perturbation conditions [Eq. (26)]:

$Z=\frac{\hat{V}(x=0)}{\hat{i}(x=0)}$

The hat $(\wedge)$ denotes small perturbation quantities. The current density is given by [Eq. (27)]:

$\hat{i}(x=0)=q A \hat{J}(x=0)$

The flux takes the form [Eq. (28)]:

$\hat{\jmath}=-D_{0} \frac{\partial \hat{n}_{c}}{\partial x}$

The voltage relates to concentration as [Eq. (29)]: 
$\hat{n}_{\mathrm{c}}(x=0)=\frac{\partial n_{\mathrm{c}}}{\partial V} \hat{V}=\frac{1}{q} c_{\mu}^{\mathrm{cb}}(x=0) \hat{V}$

To calculate the relationship of carrier flux to concentration at the left edge of the layer (Figure 1), we apply small perturbation expansion of Equation (1) [Eq. (30)]:

$\frac{\partial \hat{n}_{c}}{\partial t}=-\frac{\partial \hat{\jmath}_{n}}{\partial x}-\left(\frac{\partial U_{n}}{\partial n_{c}}\right) \hat{n}_{c}-\frac{\partial \hat{n}_{L}}{\partial t}$

Equation (30) may be completed by a kinetic equation for the traps that defines the variation $\partial n_{L} / \partial t t^{[7]}$ However, if the trapping kinetics are fast (with respect to the time scale of the transient measurement), we may assume that the traps follow the equilibrium relation with the free carriers [Eq. (31)]:

$\frac{\partial \hat{n}_{L}}{\partial t}=\frac{\partial n_{L}}{\partial n_{c}} \frac{\partial \hat{n}_{c}}{\partial t}$

Equation (31) is termed the quasistatic approximation, and it was introduced to account for the properties of measured time constants in DSCs. ${ }^{[6,7,9]}$ By applying the quasistatic approximation and the definition of the lifetime of the free carrier, Equation (30) becomes [Eq. (32)]:

$\left(1+\frac{\partial n_{L}}{\partial n_{c}}\right) \frac{\partial \hat{n}_{c}}{\partial t}=-\frac{\partial \hat{\jmath}}{\partial x}-\frac{1}{\tau_{f}} \hat{n}_{c}$

By transformation to Laplace domain $\partial / \partial t \rightarrow i \omega$, in which $\omega$ is the angular frequency of the small perturbation, we can write [Eq. (33)]:

$-\frac{\partial \hat{J}_{n}}{\partial x}=\left[i \omega \Theta_{L}+\frac{1}{\tau_{f}}\right] \hat{n}_{c}$

Let us define the quantity [Eq. (34)]:

$\Lambda_{n}(\omega, x)=D_{0}^{1 / 2}\left[i \omega \Theta_{L}+\frac{1}{\tau_{f}}\right]^{-1 / 2}$

$=L_{n}\left[1+\frac{i \omega}{\omega_{\text {rec }}}\right]^{-1 / 2}$

and we remark that [Eq. (35)]:

$\Lambda_{n}(\omega=0)=L_{n}$

Then, we express Equation (33) as [Eq. (36)]:

$\frac{\partial^{2} \hat{n}_{\mathrm{c}}}{\partial x^{2}}-\frac{\hat{n}_{\mathrm{c}}}{\Lambda_{n}(\omega, x)}=0$

Equation (36) is a classical diffusion equation; however, the coefficient $\Lambda_{n}$ is position dependent due to the $\partial n_{L} / \partial n_{\mathrm{c}}$ and $\tau_{\mathrm{f}}$ terms in Equation (34) that depend on the local value of the Fermi level. To obtain an analytical solution, let us assume that $\Lambda_{n}$ is nearly homogeneous. The solution $\hat{n}_{c}$ of Equation (36) that satisfies the boundary conditions of Equation (29) and $\hat{J}_{n}(x=L)=0$ is [Eq. (37)]:

$\hat{n}_{c}(0)=\frac{1}{q} c_{\mu}^{\mathrm{cb}} \hat{V}\left[\cosh \left(\frac{x}{\Lambda_{n}}\right)-\tanh \left(\frac{L}{\Lambda_{n}}\right) \sinh \left(\frac{x}{\Lambda_{n}}\right)\right]$

We can find the current by using Equation (27), and the impedance function is obtained [Eq. (38)]:

$Z=\frac{\Lambda_{n}(\omega)}{A D_{0} c_{\mu}^{c b}} \operatorname{cotanh}\left(\frac{\mathrm{L}}{\Lambda_{\mathrm{n}}(\omega)}\right)$

Although Equation (38) is the solution of the impedance model under the stated approximations, it is convenient to express this function in alternative forms that clarify the physical interpretation. By using the characteristic frequencies introduced in Equations (23) and (24) we can write [Eq. (39)]:

$Z=\frac{\tau_{n}}{A c_{\mu}^{\text {tot }} L_{n}}\left(1+\frac{i \omega}{\omega_{\text {rec }}}\right)^{-1 / 2} \operatorname{cotanh}\left\{\left(\frac{\omega_{\text {rec }}}{\omega_{\text {d }}}+\frac{\mathrm{i} \omega}{\omega_{\mathrm{d}}}\right)^{1 / 2}\right\}$

and also [Eq. (40)]:

$Z(\omega)=\left(\frac{R_{\mathrm{tr}} R_{\mathrm{rec}}}{1+\frac{i \omega}{\omega_{\text {rec }}}}\right)^{1 / 2} \operatorname{cotanh}\left[\left(\frac{R_{\mathrm{tr}}}{R_{\text {rec }}}\right)^{1 / 2}\left(1+\frac{i \omega}{\omega_{\text {rec }}}\right)^{1 / 2}\right]$

This result is the same as the standard expression, ${ }^{[1]}$ but now the parameters in Equation (40) are well justified with the inclusion of the trapping factors.

In connection with the last two expressions, let us define the following parameters. First, $C_{G}$ with dimensions of capacitance [F] [Eq. (41)]:

$C_{\mathrm{G}} \equiv A c_{\mu}^{\mathrm{tot}} L_{n}$

and $R_{\mathrm{G}}$ is a resistance $[\Omega]$ [Eq. (42)]:

$R_{\mathrm{G}} \equiv \frac{\tau_{n}}{C_{\mathrm{G}}}=\left(R_{\mathrm{tr}} R_{\mathrm{rec}}\right)^{1 / 2}$

These will be used below to describe the total [direct current $(D C)]$ resistance and the Gerischer impedance.

\section{Transmission-Line Model}

A transmission line (TL) is a general type of model for the propagation of a signal in space. ${ }^{[24]}$ In the electrical case, the model is defined in a 1D system with a local voltage $\hat{\varphi}$ and current $\hat{i}$ and local impedances $\chi$ and $\zeta$ [Eqs. (43) and (44)]: ${ }^{[1]}$

$\frac{\partial \hat{\varphi}}{\partial x}=-\chi(x) \hat{i}$

$\frac{\partial \hat{i}}{\partial x}=\frac{1}{\zeta(x)} \hat{\varphi}$ 
The impedance of this system can be represented as the distributed equivalent circuit shown in Figure $2 a^{[25-27]}$ Equations (43) and (44) can be taken to the form of Equation (36) to apply the method of solution indicated above. Another alternative to solve the system of $m$ equations is to establish a discrete equivalent circuit and to apply a continued fraction to sum the total impedance. ${ }^{[28]}$

a)

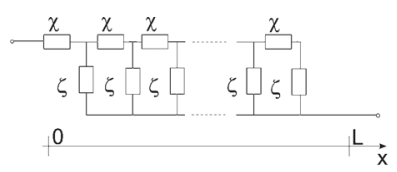

b)

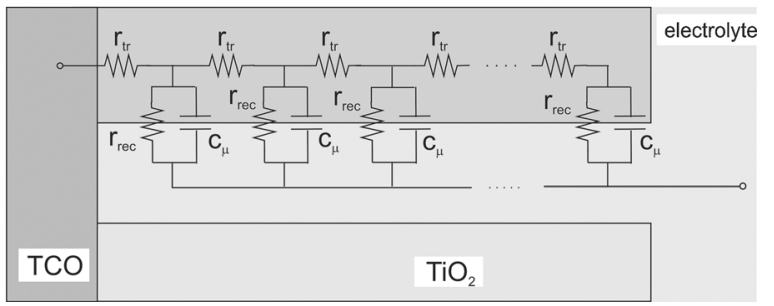

Figure 2. a) Transmission-line model with blocking boundary conditions. b) The transmission line in the diffusion-recombination model.

If the quantity $\lambda=(\zeta / \chi)^{1 / 2}$ is independent of position, then the system of differential equations can be integrated with an analytical solution [Eq. (45)]:

$Z=\lambda \chi(0) \operatorname{cotanh}(L / \lambda)$

which corresponds to Equation (38).

The equations of the diffusion-recombination model in small perturbation, discussed in the previous section, can be taken to the form of Equations (43) and (44) to show the structure of the TL. Equation (28) can be written [Eq. (46)]:

$\hat{i}=-\frac{1}{r_{\mathrm{tr}}} \frac{\partial \hat{V}}{\partial x}$

for which [Eq. (47)]:

$r_{\mathrm{tr}}=\frac{1}{A D_{n} c_{\mu}^{\mathrm{tot}}}=\frac{1}{A D_{0} c_{\mu}^{\mathrm{cb}}}=\frac{k_{\mathrm{B}} T}{A q^{2} D_{0}} n_{\mathrm{c}}^{-1}$

is a distributed transport resistance, so that we obtain $\chi=r_{\text {tr }}$ as shown in Figure 2. In Equation (47), we indicated explicitly the dependence of the local impedance element on the concentration of free carriers, $n_{c}$.

The equation for spatial variation of the current [Eq. (33)] can be stated as [Eq. (48)]:

$\frac{\partial \hat{i}}{\partial x}=\left[\frac{1}{r_{\text {rec }}}+i \omega A c_{\mu}^{\mathrm{tot}}\right] \hat{V}$
In this equation, we have introduced a distributed recombination resistance [Eq. (49)]:

$r_{\text {rec }}=\frac{\tau_{n}}{A c_{\mu}^{\text {tot }}}=\frac{\tau_{f}}{A c_{\mu}^{c b}}$

and clearly, $A c_{\mu}^{\text {tot }}$ is the chemical capacitance per unit length. The transverse element of the TL is therefore given by [Eq. (50)]:

$\zeta=\left[\frac{1}{r_{\text {rec }}}+i \omega A c_{\mu}^{\text {tot }}\right]^{-1}$

The full transmission-line model for diffusion-recombination is shown in Figure $2 \mathrm{~b}$.

We note that the local impedances $r_{\text {tr }}$ and $r_{\text {rec }}$ depend only on the quantities of the free carriers. Indeed, the resistances are not affected by trapping factors in the multiple trapping model. However, any time-dependent measurement involves a capacitive component, and it is unavoidable to measure $A c_{\mu}^{\text {tot }}$, which contains the contribution from the traps. Thus, the total capacitance appears in the impedance as indicated in Equation (50), and this will also be reflected in the measurement of any time constant by whatever technique, as mentioned above.

We can find from Equations (47) and (50) that [Eq. (51)]:

$\lambda=\left(\zeta / r_{\mathrm{tr}}\right)^{1 / 2}=\left[\frac{1}{L_{n}^{2}}+\frac{i \omega}{D_{n}}\right]^{-1}$

We mentioned above that the condition of integrability of the TL model was that $\lambda$ must be constant. In Equation (22), we observe that $L_{n}$ is constant only for linear recombination, in which $\tau_{\mathrm{f}}=\tau_{0}$. In Equation (17), we observe that $D_{n}$ is a constant only if the traps can be neglected $\left(\Theta_{L}=1\right)$. Under these conditions, or if the quantities $\tau_{\mathrm{f}}$ and $\Theta_{L}$ can be considered uniform, Equations (38) and (45) are valid; otherwise, the impedance should be calculated numerically.

If the resistances $r_{\text {tr }}$ and $r_{\text {rec }}$ are homogeneous, then we obtain the total resistances defined above as [Eqs. (52) and (53)]:

$R_{\mathrm{tr}}=L r_{\mathrm{tr}}$

$R_{\text {rec }}=\frac{r_{\text {rec }}}{L}$

\section{Limiting Cases of DC Resistance According to Diffusion Length}

The DC resistance is a very important impedance parameter [Eq. (54)]:

$R_{\mathrm{DC}}=Z(\omega=0)$

From Equation (39) we obtain [Eq. (55)]:

$R_{\mathrm{DC}}=R_{\mathrm{G}} \operatorname{cotanh}\left(\frac{\mathrm{L}}{\mathrm{L}_{\mathrm{n}}}\right)$ 


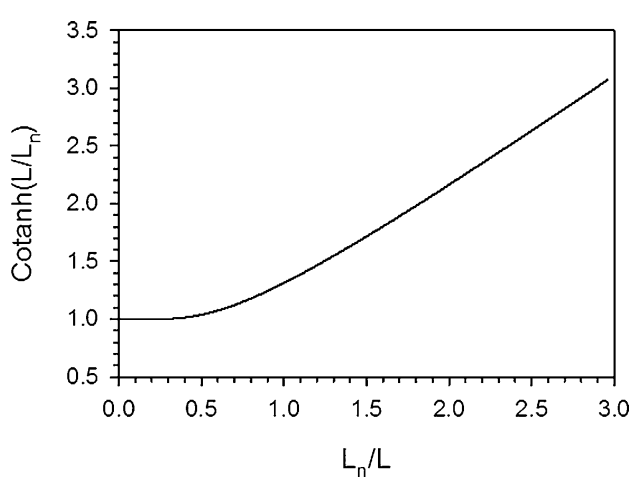

Figure 3. Representation of the function $\operatorname{cotanh}(1 / z)$.

The DC resistance takes different limiting forms depending on the parameter $z=L_{n} / L$. The function $\operatorname{cotanh}(1 / z)$ is shown in Figure 3. For the long diffusion length $\operatorname{cotanh}(1 / z) \approx z$; hence [Eq. (56)]:

$R_{\mathrm{DC}} \approx \frac{\tau_{n}}{A c_{\mu}^{\text {tot } L}}=R_{\text {rec }}$

whereas for the short diffusion length [Eq. (57)]:

$R_{\mathrm{DC}} \approx \frac{\tau_{n}}{A c_{\mu}^{\text {tot }} L_{n}}=R_{G}$

$R_{\mathrm{DC}}$ does not depend on thickness in the case of the short diffusion length.

\section{Interpretation of Diffusion-Recombination Impedance}

Equation (40) is one way to write the diffusion-recombination impedance, but of course, one can use other sets of parameters: the chemical capacitance, the chemical diffusion coefficient, and so on by using the expressions given above. Reparametrization has sometimes led to confusion regarding the information that can be obtained from the impedance spectra.

The diffusion-recombination impedance provides two basic kinds of spectra according to the conditions of Equation (20). The case of a long diffusion length, that is, the spectrum for which $R_{\mathrm{tr}}<R_{\text {rec }}$ is shown in Figure $4 \mathrm{a}$, b. At high frequency, it contains the characteristic Warburg impedance of diffusion, with an inclination of $45^{\circ}$, which turns into an arc at low frequency. The arc is given by the standard expression [Eq. (58)]:[1]

$Z=\frac{1}{3} R_{\mathrm{tr}}+\frac{R_{\mathrm{rec}}}{1+\frac{i \omega}{\omega_{\mathrm{rec}}}}$

If the resistances $R_{\mathrm{tr}}$ and $R_{\text {rec }}$ can be determined from the spectra, then the diffusion length can be obtained from the expression derived from Equation (25) [Eq. (59)]: ${ }^{[1]}$

$L_{n}=\left(\frac{R_{\mathrm{rec}}}{R_{\mathrm{tr}}}\right)^{1 / 2} L$ (a)

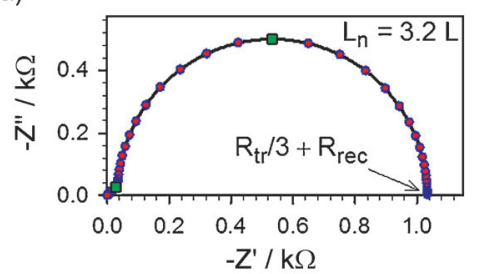

(b)

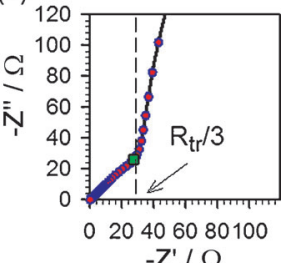

(c)
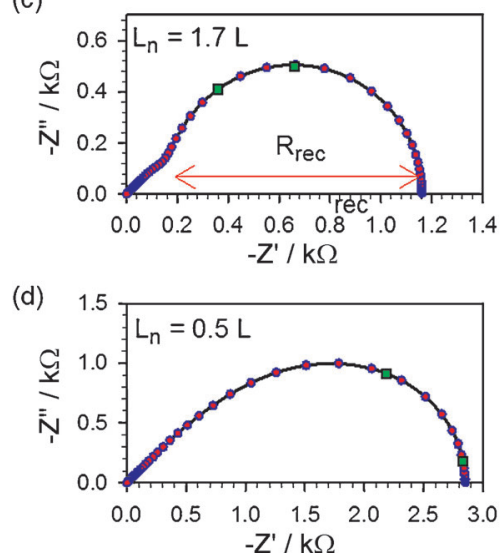

Figure 4. a) Diffusion-recombination impedance spectra with reflecting boundary conditions. Simulation of the impedance with parameters $R_{\text {rec }}=10^{3} \Omega, C_{\mu}=5 \times 10^{-6} \mathrm{~F}$, and increasing transport resistance $R_{\mathrm{tr}}=10^{2}$ $(\mathrm{a}, \mathrm{b}), R_{\mathrm{tr}}=10^{3}(\mathrm{c})$, and $R_{\mathrm{tr}}=10^{4} \Omega(\mathrm{d})$. The frequency $[\mathrm{Hz}]$ at selected points is shown: the characteristic frequency of the low-frequency arc $(\mathbf{\square})$, related to the angular frequency $\omega_{\text {rec }}=\tau_{n}^{-1}$, and the low-frequency resistance. The frequency $[\mathrm{Hz}]$ of the turnover from Warburg behavior to the low-frequency recombination $\operatorname{arc}(\mathbf{m})$, related to the characteristic frequency $\omega_{\mathrm{d}}$, is also shown.

If the diffusion Warburg part is indeed detected, the impedance model provides three basic parameters: the recombination resistance, $R_{\text {reci }}$ the chemical capacitance, $C_{\mu}^{\text {tot }}$; and the transport resistance, $R_{\mathrm{tr}}$. Regardless of the specific parameters used for fitting, there are three independent impedance parameters that can be derived from the model. However, if the conductivity is large, the transport resistance vanishes and cannot be observed. Only the arc in Figure $4 \mathrm{a}$ will be observed, and the only information that can be derived from the spectrum is recombination resistance, $R_{\text {rec }} ;$ the chemical capacitance, $C_{\mu}^{\text {tot }}$; and parameters derived from these two. No parameter of transport can then be obtained from the measurement.

\section{The Gerischer Impedance}

In the case of strong recombination, the diffusion length becomes short $\left(L_{n}<L\right)$. The injected carriers penetrate to a restricted extent into the layer, of the order $L_{n}$ (Figure 1), so that all the impedance parameters should be independent on the actual thickness $L$. The impedance spectra adopt the form of the Gerischer impedance [Eq. (60)]:

$Z=\frac{R_{\mathrm{G}}}{\left(1+\frac{i \omega}{\omega_{\mathrm{rec}}}\right)^{1 / 2}}$ 

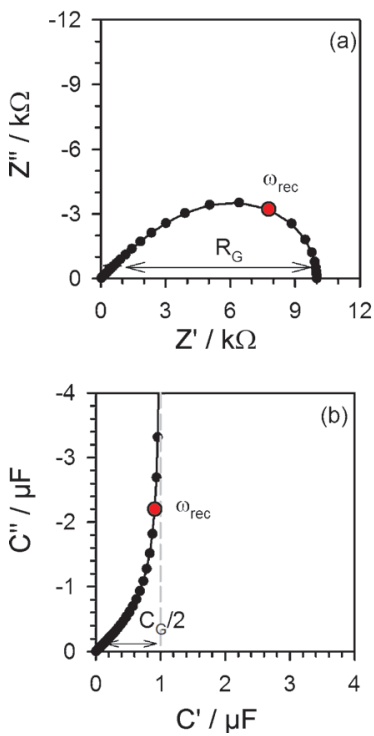
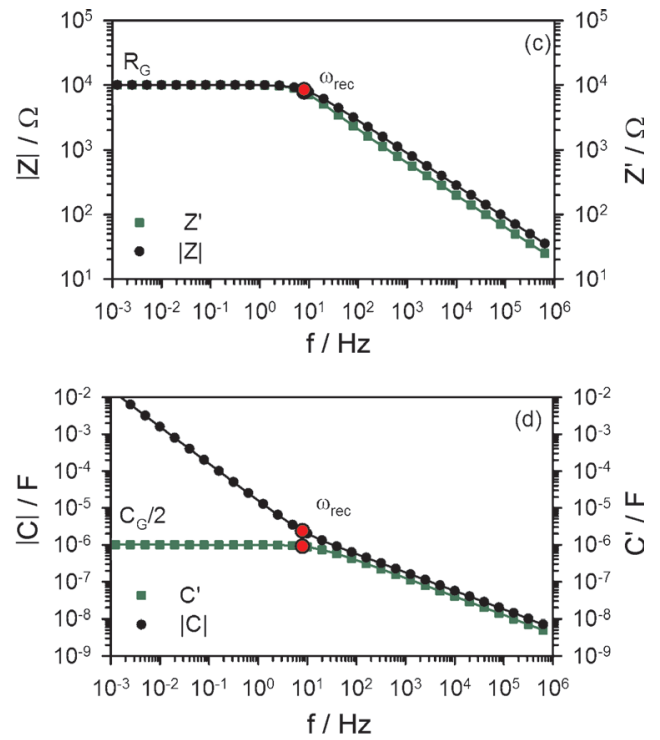

Figure 5. Simulated Gerischer impedance with $R_{\mathrm{G}}=10 \mathrm{k} \Omega, \omega_{\mathrm{rec}}=50 \mathrm{rads}^{-1}$, and $C_{\mathrm{G}}=1 \mu \mathrm{F}$. Nyquist $(\mathrm{a}, \mathrm{b})$ and bode $(c, d)$ diagrams of impedance $(a, c)$ and capacitance $(b, d)$.

shown in Figure $4 \mathrm{~d}$ and in several different representations in Figure 5. In Equation (42), $R_{\mathrm{tr}}$ and $R_{\text {rec }}$ appear in $R_{\mathrm{G}}$ as a product and cannot be separately determined. ${ }^{[16]}$ In Section 5, it was shown that $R_{\mathrm{DC}}$ follows $R_{\text {rec }}$ at low applied voltage and coincides with $R_{\mathrm{G}}$ after the cross-over potential.

The square root of the resistances in Equation (42) depends only on volume-specific quantities but not on film thickness $L$, as mentioned above, and we expect Equation (60) to depend on the diffusion length.

The impedance in Equation (60) can be expressed as [Eq. (61)]:

$Z=\frac{1}{C_{G} \omega_{\text {rec }}} \frac{1}{\left(1+\frac{i \omega}{\omega_{\text {rec }}}\right)^{1 / 2}}$

Thus, the Gerischer impedance provides two independent parameters, $C_{G}$ (which contains the diffusion length) and $\omega_{\text {rec }}$. Of course, other choices can be proposed, but it is not possible to determine three separate parameters, as in Equation (40).

From Equation (61), we may write the frequency-dependent Gerischer capacitance as [Eq. (62)]:

$C^{*}(\omega)=\frac{1}{i \omega Z}=C_{\mathrm{G}} \frac{\omega_{\mathrm{rec}}}{i \omega}\left(1+\frac{i \omega}{\omega_{\mathrm{rec}}}\right)^{1 / 2}$

which at low frequency yields [Eq. (63)]:

$C_{\mathrm{If}}=\frac{C_{\mathrm{G}}}{2}\left(1-i \frac{\omega_{\mathrm{rec}}}{\omega}\right)$

There are several different parameters to characterize the Gerischer impedance (see the Supporting Information). A direct advantage that we may obtain from the representation in Equation (61) is that it is possible to read the values of $R_{\mathrm{G}}$,
$C_{G}$, and $\omega_{\text {rec }}$ from the complex plane plots and bode plots, as shown in Figure 5. In particular, $R_{\mathrm{G}}$ represents the DC limit of the Gerischer impedance, whereas $C_{G} / 2$ represents the $\mathrm{DC}$ limit of the real part of the Gerischer capacitance.

\section{Discussion}

In experimental situations, the impedance spectra of a solar cell are determined over a wide range of voltages. ${ }^{[2,3,13-15]}$ One may expect to obtain $C_{\mu}, R_{\text {rec }}$ and $R_{\mathrm{tr}}$ if $L_{n}>L$, but if the variation of voltage causes $L_{n}<L$, then only $\tau_{n}$ and $C_{G}$ can be determined. In practice, $L_{n} \approx 0.5 \mathrm{~L}$ is the limit for obtaining all three parameters.

Equation (41) shows that the diffusion length is available from the Gerischer impedance only if the specific chemical capacitance can be determined. It is not possible to obtain the chemical capacitance from the impedance data if $L_{n}<L$, but it can be extrapolated from the voltage domain where $L_{n}>L$, for which the general expression given in Equation (40) applies. In contrast, $C_{G}$ can be determined over the whole voltage range, and it should be compared to $C_{\mu}^{\text {tot }}$.

As an application of the analytical theory, we consider a model system that is trap free $\left(\Theta_{L}=1, D_{n}=D_{0}, c_{\mu}^{\text {tot }}=c_{\mu}^{\mathrm{cb}}\right)$ but with nonlinear recombination that produces a lifetime that decreases with the voltage, as indicated by the following expressions [Eqs. (64), (65), and (66)]:

$n_{\mathrm{c}}=n_{0} e^{q V / k_{\mathrm{B}} T}$

$c_{\mu}^{\text {tot }}=\frac{n_{0} q^{2}}{k_{\mathrm{B}} T} e^{q V / k_{\mathrm{B}} T}$

$\tau_{n}=\tau_{f}=\tau_{0} e^{-q \gamma V / k_{B} T}$

Thus, the diffusion length decreases with increasing voltage [Eq. (67)]:

$L_{n}=L_{0} e^{-q \gamma V / 2 k_{B} T}$

in which $L_{0}=\sqrt{D_{0} \tau_{0}}$. In this case, from Equations (11), (61), and (62) we obtain [Eq. (68)]:

$r_{\text {rec }}=\frac{k_{B} T}{q^{2}} \frac{\tau_{0}}{n_{0}} e^{-q(1+\gamma) V / k_{B} T}$

The results of the calculations of the different parameters introduced above are shown in Figure 6 . We remark the crossover of the parameters at the voltage at which $L_{n}=L$. 

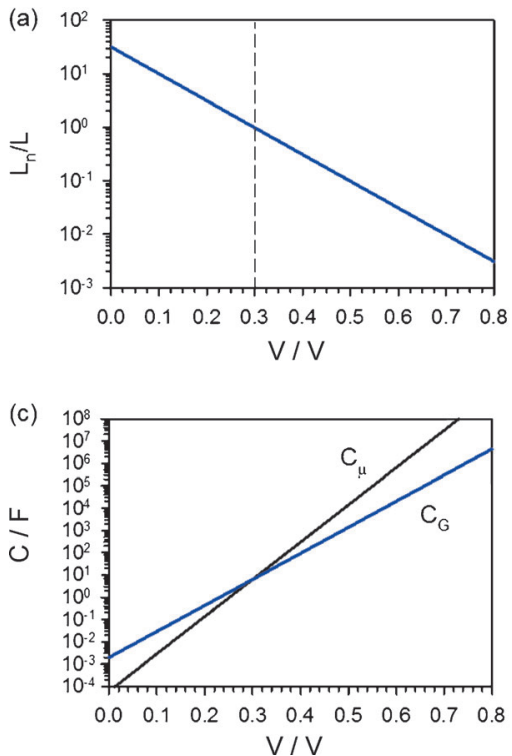
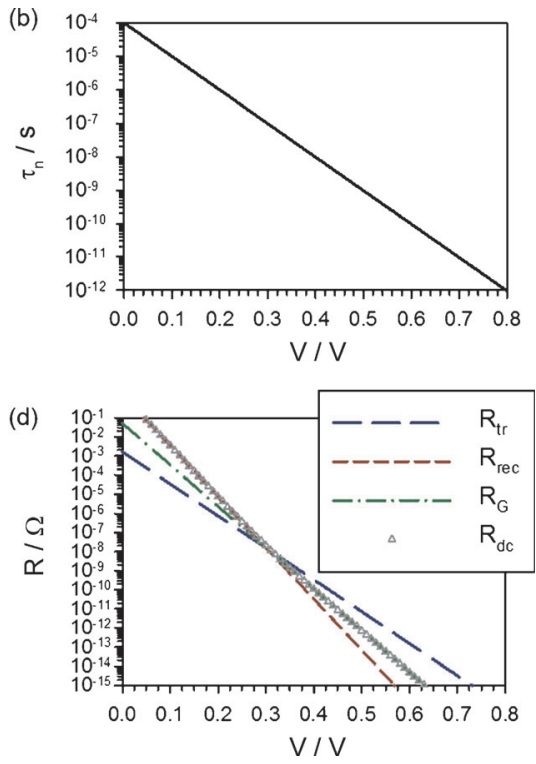

Figure 6. Representation of impedance parameters of a trap-free model system with the following values: $L=10 \mu \mathrm{m}, A=1 \mathrm{~cm}^{2}, n_{0}=10^{16} \mathrm{~cm}^{-3}, D_{0}=10 \mathrm{~cm}^{2} \mathrm{~s}^{-1}, \tau_{0}=10^{-4} \mathrm{~s}$, and $\gamma=0.6$.

[1] J. Bisquert, J. Phys. Chem. B 2002, $106,325-333$.

[2] Q. Wang, S. Ito, M. Grätzel, F. Fabregat-Santiago, I. Mora-Seró, J. Bisquert, T. Bessho, H. Imai, J. Phys. Chem. B 2006, 110, 19406-19411.

[3] F. Fabregat-Santiago, G. Garcia-Belmonte, I. Mora-Seró, J. Bisquert, Phys. Chem. Chem. Phys. 2011, 13, 9083-9118.

[4] J. Bisquert, F. Fabregat-Santiago in Impedance Spectroscopy: A General Introduction and Application to Dye-Sensitized Solar Cells (Ed.: K. Kalyanasundaram), CRC, Boca Raton, 2010.

[5] J. Bisquert, R. A. Marcus, Top. Curr. Chem., 2013, DOI: 10.1007/1128_ 2013_1471.

[6] J. Bisquert, V. S. Vikhrenko, J. Phys. Chem. B 2004, 108, 2313-2322.

[7] J. Bisquert, Phys. Rev. B 2008, 77, 235203.

[8] J. Bisquert, Phys. Chem. Chem. Phys. 2008, 10, 3175-3194.

[9] M. Ansari-Rad, J. A. Anta, J. Bisquert, J. Phys. Chem. C 2013, 117, $16275-16289$.

\section{Summary and Conclusions}

The characterization of semiconductor solar cells by impedance spectroscopy provides a number of parameters: the chemical capacitance, $C_{\mu}$; the recombination resistance, $R_{\text {reci }}$; the transport resistance, $R_{\mathrm{tr}}$; the electron lifetime, $\tau_{n}$; the electron conductivity, $\sigma_{n}$; the chemical diffusion coefficient, $D_{n}$; and the diffusion length, $L_{n}$. Of this collection, at most only three are independent parameters that can be used to fit the impedance spectra. However, in some cases not all such parameters can be determined. If transport is very efficient, $R_{\mathrm{tr}}$ cannot be measured. The number of fitting parameters is reduced to two, and the transport parameters $\sigma_{n}, D_{n}$, and $L_{n}$ cannot be determined. In contrast, if the diffusion length is short, then $R_{\text {rec }}<R_{\mathrm{tr}}$. In this case, the number of parameters is reduced to two in the Gerischer impedance. We showed that one parameterization consists of the lifetime, $\tau_{n}$, and the capacitance $C_{G}=A c_{\mu}^{\text {tot }} L_{n}$, which in essence implies that the chemical capacitance of the semiconductor film is taken over one diffusion length. These remarks should be useful for the analysis of impedance spectra of solar cells.

\section{Acknowledgements}

We acknowledge support by Generalitat Valenciana (PROMETEO/ 2009/058). We are grateful to Luca Bertoluzzi for discussions.

Keywords: conducting materials - electrochemistry impedance spectroscopy $\cdot$ nanostructures $\cdot$ photovoltaics
[10] G. Garcia-Belmonte, A. Guerrero, J. Bisquert, J. Phys. Chem. Lett. 2013, 4, $877-886$

[11] J. A. Anta, J. Idigoras, E. Guillen, J. Villanueva-Cab, H. J. Mandujano-Ramirez, G. Oskam, L. Pelleja, E. Palomares, Phys. Chem. Chem. Phys. 2012, 14, 10285-10299.

[12] J. Bisquert, I. Mora-Seró, J. Phys. Chem. Lett. 2010, 1, 450-456.

[13] J. R. Jennings, F. Li, Q. Wang, J. Phys. Chem. C 2010, 114, 14665-14674.

[14] S. R. Raga, E. M. Barea, F. Fabregat-Santiago, J. Phys. Chem. Lett. 2012, 3, $1629-1634$.

[15] J. R. Jennings, Y. Liu, F. Safari-Alamuti, Q. Wang, J. Phys. Chem. C 2012, $116,1556-1562$.

[16] Y. Liu, J. R. Jennings, Y. Huang, Q. Wang, S. M. Zakeeruddin, M. Grätzel, J. Phys. Chem. C 2011, 115, 18847-18855.

[17] J. P. Gonzalez-Vazquez, J. A. Anta, J. Bisquert, Phys. Chem. Chem. Phys. 2009, 11, 10359-10367.

[18] J. Bisquert, Phys. Chem. Chem. Phys. 2003, 5, 5360-5364.

[19] J. Bisquert, F. Fabregat-Santiago, I. Mora-Seró, G. Garcia-Belmonte, S. Giménez, J. Phys. Chem. C 2009, 113, 17278-17290.

[20] J. Chen, B. Li, J. Zheng, S. Jia, J. Zhao, H. Jing, Z. Zhu, J. Phys. Chem. C 2011, 115, 7104-7113.

[21] H. Wang, L. M. Peter, J. Phys. Chem. C 2009, 113, 18125-18133.

[22] J. Villanueva-Cab, H. Wang, G. Oskam, L. M. Peter, J. Phys. Chem. Lett. 2010, 1, 748-751.

[23] J. P. Gonzalez-Vazquez, G. Oskam, J. A. Anta, J. Phys. Chem. C 2012, 116, $22687-22697$.

[24] L. Brioullin, Wave Propagation in Periodic Structures, Dover, New York, 1953.

[25] J. Bisquert, M. Grätzel, Q. Wang, F. Fabregat-Santiago, J. Phys. Chem. B 2006, 110, 11284-11290.

[26] W. Shockley, Proc. IRE 1958, 46, 973-990.

[27] C.-T. Sah, Proc. IEEE 1967, 55, 654.

[28] J. C. Wang, J. Electrochem. Soc. 1987, 134, 1915-1920.
Received: July 5, 2013

Published online on $\mathbf{\square}$ II, 2013 


\section{FULL PAPERS}

Diffusing out: The theory of diffusionrecombination impedance applied to nanostructured solar cells is presented with an emphasis on the effects of energy disorder and short diffusion length.
Diffusion-recombination impedance

$\bar{N}$

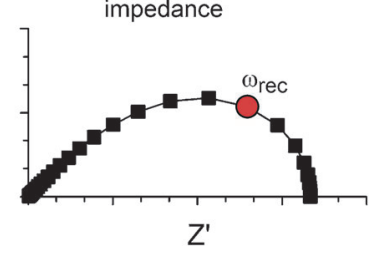

J. Bisquert, ${ }^{*}$ I. Mora-Sero,

F. Fabregat-Santiago

Diffusion-Recombination Impedance Model for Solar Cells with Disorder and Nonlinear Recombination 